\title{
Reshaping Drug Development using 3D Printing
}

Atheer Awad ${ }^{1 *}$, Sarah J. Trenfield ${ }^{1 *}$, Alvaro Goyanes ${ }^{2}$, Simon Gaisford ${ }^{1,2}$ and Abdul W. Basit ${ }^{1,2}$.

*Both authors contributed equally to this work

1UCL School of Pharmacy, University College London, 29-39 Brunswick Square, London, WC1N 1AX, UK

${ }^{2}$ FabRx Ltd., 3 Romney Road, Ashford, Kent, TN24 0RW, UK

Corresponding author: Basit, A.W.

(a.basit@ucl.ac.uk)

Tel: 02077535865

\section{Key words}

Additive manufacturing; three-dimensional printing; rapid prototyping; drug discovery; industrial revolution; pharmaceuticals

\section{5-30 word teaser}

This review aims to overview the potential for 3D printing to trigger a paradigm shift in the pharmaceutical sector, offering a forward look across drug development, manufacturing and supply. 


\section{Abstract}

The pharmaceutical industry stands on the brink of a revolution, calling for the recognition and embracement of novel techniques. Three-dimensional printing (3DP) is forecast to reshape the way medications are designed, manufactured and used. Whilst a clear trend towards personalised fabrication is perceived, this review aims to accentuate the merits and shortcomings of each technology, providing an insight on aspects such as efficiency of production, global supply and logistics. Contemporary opportunities of 3DP in drug discovery and pharmaceutical development and manufacturing are unveiled, offering a forward-looking view on its potential uses as a digitised tool for personalised dispensing of medicines. 


\subsection{Introduction}

Three-dimensional printing (3DP) has the potential to cause a paradigm shift in the way that medicines are designed, manufactured and used. For centuries, civilisation has experienced periodic radical transformations, often described as industrial revolutions. With the advent of steam engines, the textile industry and mechanised factories, the first industrial revolution was pronounced [1]. Motivated by the harnessing of electrical energy for mass production, the second industrial revolution evolved [2]. Subsequently, the third industrial revolution was established by the adoption of automation [3]. Robotised and customised systems, such as cloud computing, the internet of things (IOT) and 3DP, have already been implemented to bridge the gap between the physical and virtual worlds [4]. Now, 3DP is at the forefront of the next industrial revolution.

3DP has created a technological paradigm by triggering boundless opportunities in diverse fields. It is an additive manufacturing technique that enables the fabrication of bespoke objects in a layered manner. By combining digitisation and mechanisation, this disruptive tool avoids the constraints often imposed by conventional tooling methods. Owing to its additive nature, 3DP delivers finalised products rapidly, with minimal waste production [5]. Additionally, as the object designs are digitised, their customisation, storage and transference can be achieved with ease, avoiding the need for labour and space occupancy. Collectively, this permits the instantaneous creation of complex bespoke objects with ease. Thus, this singular platform has a multitude of applications ranging from aviation to automobiles, medicine, dentistry, art, jewellery, and footwear [6].

In the pharmaceutical field, the drug development process is a multistage procedure, requiring a great deal of resources and time. Since the 1960s, this sector has been experiencing a dormant stage, whereby limited manufacturing advancements have been made. Recently, 3DP has offered contemporary opportunities to revolutionise the pharmaceutical industry. In particular, 3DP can be used to fabricate 'printlets', which is a term that refers 
to 3D printed solid oral dosage forms (e.g. tablets and capsules). As such, this multidisciplinary tool could be implemented in all the drug development stages, enhancing the quality of treatment in healthcare.

Whilst most research in this area is primarily focused on personalised medicines, a multitude of opportunities remain underexplored (Figure 1). In our previous review, we discussed the motivations and potential applications of 3DP in clinical research and practice, providing a practical viewpoint on its integration in a pharmaceutical setting, whilst highlighting the challenges and hurdles that come alongside [7]. In this review, we focus on the technical aspects, offering an overview on the novel prospects via which 3DP can be applied to the different drug development phases, including its discovery, early screening, testing, manufacturing, and dispensing, while discussing some of the technological hurdles associated with the adoption of these processing for pharmaceutical production.

Insert figure 1.

\subsection{D Printing to Support Drug Discovery}

It is well understood that the drug failure rate is high during early phase development [8], creating a substantial financial burden for the pharmaceutical industry. In 2013, the cost of taking a new chemical entity to commercialisation was estimated at $\sim \$ 5$ billion dollars (Herper, M., The Cost of Creating a New Drug Now \$5 Billion, Pushing Big Pharma to Change, https://http://www.forbes.com/sites/matthewherper/2013/08/11/how-thestaggering-cost-of-inventing-new-drugs-is-shaping-the-future-of-medicine/ 131da9b13c33; 2013, [accessed 06 February 2018]), and this value will only continue to increase over the next decade. As such, there is a growing need for innovative technologies to support drug development, in particular, by enabling a rapid identification of suitable drug candidates at a minimal cost. 3DP could prove advantageous for this application by producing small or 'oneoff' batches of formulations (and even drugs) in a cost-effective, efficient and 
flexible manner. Using such technology could expedite the drug development process.

Early phase drug development covers the fields of drug discovery, pre-clinical studies and first-in-human $(\mathrm{FIH})$ clinical trials. Within drug discovery, 3DP has already been used to produce active pharmaceutical ingredients (APIs). Chemists from the University of Glasgow fabricated a series of reaction vessels (composed of polypropylene) using fused deposition modelling (FDM). The RepRap printer used was modified to incorporate liquid handling components, whereby liquid reagents could be dispensed into the reaction vessels after fabrication to carry out simple chemical reactions on a small scale [9]. Thus far, the group has produced ibuprofen and aim to make other molecules using this novel approach. Further to this, Kitson et al. [10] successfully undertook the multistep synthesis of baclofen within a 3D printed miniaturised reactor cascade, thus demonstrating the ability of 3DP to remotely digitise blueprints for print and synthesis.

3DP of miniaturised reaction vessels for API synthesis on demand could provide a greater deal of flexibility to scientists. Compared with conventional methods, 3DP could help to support the synthesis of a range of different molecules on a small scale, particularly useful for those of high cost or poor stability [11]. Moreover, it could enable researchers to evaluate different chemical reactions and reaction conditions, enabling synthesis pathways to be more efficiently established. Printing reaction vessels on demand could also enable API synthesis to be performed at locations that could otherwise not support such processes, such as within remote locations or even for expensive personalised medicines in the clinic. However, there are limitations to the process, including consideration of solvent incompatibilities and heat tolerance of the printed materials, requiring more research to be performed in this area before integration [12]. Other considerations surrounding decentralising production are discussed in Section 4.0.

These benefits could also be extended to the field of pre-clinical drug development. Through advancements in bioprinting, researchers have been 
able to 3D print animal and human tissues, which could be suitable for acute and chronic drug toxicity screening, as well as metabolic studies. For example, Organovo specialises in 3D bioprinting of structurally and functionally accurate human tissue models (such as liver and kidney tissue) that can be used for medical and therapeutic research (Organovo, Changing the shape of medical research and practice: Structurally and functionally accurate bioprinted human tissue models, http://organovo.com; [accessed 06 February 2018]). Moreover, 3DP has been used to create 'organs-on-a-chip', designed to mimic the structure and function of human or even diseased tissue. Researchers at Harvard University 3D printed the first cardiac microphysiological device, which was used to study drug responses as well as contractile development of laminar cardiac tissues [13]. This has also been taken a step further, whereby a variety of organ models have been $3 \mathrm{D}$ bioprinted, ranging from the pancreas (Pancreas from PLA and Human Stem Cells, https://3dprint.com/157430/3d-printed-pancreas-celprogen/ 2016, [accessed 08 February 2018]), to the stomach and small intestine [14]. As such, 3DP could open up new avenues for in vitro testing of drug response and toxicology screening. In particular, more effective biorelevant models could be created, improving the accuracy of new drug candidate screening. If such biorelevant models were to be developed using 3DP, in the future this could reduce the number of animals required for pre-clinical studies, reducing costs of development and time-to-market [15].

During formulation development, on demand 3DP could be used to produce several drug product iterations to evaluate dosage form suitability, both within animal models and humans $[16,17]$. In particular, one-off or small batches of printlets, each with different formulation compositions, could be produced rapidly, streamlining the evaluation of attributes such as excipient inclusion, compatibility and drug performance within in vitro and in vivo models. As such, compared to more lengthy manufacturing technologies, 3DP could enable an earlier collection of information required for clinical go/no-go decisions, in turn expediting entry into $\mathrm{FIH}$ clinical trials to reduce time and cost of development [7]. 
Moreover, it is well known that pre-clinical development and FIH trials require evaluation of a wide dose range [18]. Due to the flexibility of 3DP, a wide range of dosages and geometries could be created to suit the study requirements [19-21]. As such, compared to traditional manufacturing processes, the development of an optimal product could occur more swiftly without increasing lead-times or development costs (Stratasys, How 3D Printing Will Continue to Transform Manufacturing, https://http://www.stratasysdirect.com/content/white papers/str 746315 sd m wp transform mfg.pdf;. [accessed 08 February 2018]). However, it is clear that current 3D printers are not amenable for scale up and, as such, considerations around how formulations could be transitioned from small to large batches (e.g. for later phase trials) require further evaluation.

\subsection{Revolutionising Drug Manufacture using 3D Printing}

Over the years, different 3DP technologies have been developed, with each possessing a unique set of attributes (Figure 2). Based on the American Society for Testing and Materials (ASTM) International, the different 3DP technologies can be classified into seven main categories [22]:

- Vat photopolymerisation; which is a process that utilises a light source (e.g. laser) to selectively cure a vat of liquid photopolymer, transforming it into a solid object. Examples of such are the stereolithography (SLA), digital light processing (DLP) and continuous liquid interface production (CLIP) technologies.

- Binder jetting (BJ); which revolves around the selective binding of solid powder particles by spraying a liquid agent.

- Powder bed fusion; which is a selective thermal process that involves the fusion of powder particles by the application of a laser or other heat source. It includes selective laser sintering (SLS), multi jet fusion 
(MJF), direct metal laser sintering/selective laser melting (DMLS/SLM) and electron beam melting (EBM).

- Material jetting; which is a selective technique in which liquid droplets of materials are deposited on a surface. These droplets spontaneously solidify (known as drop-on-demand (DOD)) or can be cured or fused using a UV light (known as material jetting (MJ)) or a heat source (known as nanoparticle jetting (NPJ).

- Direct energy deposition; which is a process that selectively deposits a form of focused thermal energy (e.g. laser) directly onto powder particles, causing them to melt and fuse. It involves two technologies; laser engineering net shape (LENS) and electron beam additive manufacturing (EBAM).

- Sheet lamination; which compromises the bonding of materials in the form of sheets (e.g. cut paper, plastic or metal) to fabricate 3D objects. It is often known as laminated object manufacturing (LOM) or ultrasonic additive manufacturing (UAM).

- Material extrusion; which is a technology that involves the selective dispensing of material in a semi-solid form. This technology is further subdivided into fused deposition modelling (FDM), which utilises thermoplastics, and semi-solid extrusion (SSE), which utilises gels and pastes.

Insert figure 2

Whilst the 3DP technologies share some common features with one another and with other manufacturing technologies, such as injection moulding, the type of final products they are capable of fabricating differ inherently. As such, Table 1 provides an overview on the features associated with the most commonly used manufacturing technologies. 
It is indeed clear that based on each technology's merits and/or demerits, its suitability to be implemented as a pharmaceutical manufacturing platform differs. For instance, as shown in table 1, perhaps the high prices of some printers (e.g. EBM, MJP, NJP, DMLS/SLM, EBAM, LENS and UAM) is one of their main shortcoming, resulting in the absence of their use in pharmaceutical research. However, in a case study involving injection moulding, it has shown that for it to be considered cost-effective, the number of produced units should exceed a certain limit [23]. This limit is however, variable and is dependent upon multiple factors, including the size of the product, the build material and the number of moulds needed. Wherein, the number of moulds will vary depending on their lifetime, which in turn depends on their quality (e.g. material they are made from) and usage (e.g. number of uses and labour handling). As such, below the abovementioned limit, the cost of customising moulds exceeds the profit gained from fabricating the products. Hence, this finding highlights the main downside associated with the use of this technology. The 3DP technologies on the other hand do not necessitate the modification of tooling or require major labouring, which can possibly compensate for the high equipment pricing.

Similarly, whilst vat polymerisation and MJ processes are characterised with high precision and speed, they share two common demerits; high cost and their potential to cause toxicity due to the presence of unreacted monomers [24]. Thus, for example, although the CLIP technology might be an ideal substitute for conventional tableting machines in terms of production speed, wherein it is capable of producing a tablet within seconds (this calculation is based on the fact that it takes CLIP $\sim 6.5 \mathrm{~min}$ to produce the same object that takes $\sim 3.5 \mathrm{hr}$ and $\sim 11.5 \mathrm{hr}$ to be produced using SLS and SLA, respectively (3Dprinting.com. Carbon3D Reaches Incredible 3D Printing Speeds with CLIP, https://3dprinting.com/news/carbon3d-reaches-incredible-3d-printingspeeds-with-clip/; 2018 [accessed 17 April 2018]). Thus, if it takes SLS and SLA 3-5 min to produce one tablet, the CLIP is expected to take a couple of seconds). Nonetheless, in pharmaceutical research, its exploitation is limited to one application so far [25]. In contrast, whilst BJ technologies utilise 
generally regarded as safe (GRAS) excipients, their final products are characterised for having low mechanical properties (e.g. low friability and hardness values) [26]. As such, their applications remain limited to certain dosage forms, where strong mechanical properties is not a requirement.

FDM suffers from the potential risk of drug degradation due to the elevated temperatures associated with the process [27]. Favourably, SSE functions in the absence of laser beams and at lowered temperatures, avoiding such hazards. Nonetheless, this technology's low resolution and mechanical properties restrict its applications to particular dosage forms, wherein complexity is not critical [28]. On the contrary, this adds to the merits of FDM, SLS and SLA, wherein almost any dosage form can be fabricated. This remarkable attribute is deemed as unique, as most conventional manufacturing methods are confined to the production of a limited type/s of dosage forms $[7,29]$.

Thus far, only a few 3DP technologies have been investigated in pharmaceutical research with FDM being the most studied technique. This high prevalence is mostly attributed to the low costs and high availability of the printers. Conversely, in a case study where the unit prices were calculated using the same reference object, SLS, SLA, FDM and injection moulding costs were compared (Figure 3) [30]. Conclusively, all the 3DP technologies were found to result in a constant unit price throughout the production process, in which SLS was the most cost-efficient. Injection moulding on the other hand, provided a quantity-dependent curve, whereby below 2,000 production units, the cost was $\sim 6-15$ fold higher than that of the 3DP technologies. This is mainly explained by the need for moulds in the case of injection moulding, wherein producing these moulds will require extra machinery, material and labour, hence the reason behind the increased cost. Additionally, an important element, namely time, plays a major role in this scenario. As such, accelerating the speed of production results in the reduction of labour, resources and energy consumption, all of which collectively reduce costs. This provides sufficient proof that the machinery 
cost solely does not provide a summative evaluation to predict profit. In fact, currently, most injection moulding moulds are produced using 3DP, as it was found to reduce the total costs by $\sim 24$ fold (Redwood, B. 3D Printing low-run injection molds, https://www.3dhubs.com/knowledge-base/3d-printing-low-runinjection-molds; 2018 [accessed 02 February 2018]). Besides, it should be pointed out, that the abovementioned cost would further increase if the produced product is modified or changed (e.g. change in shape or size), as it will require the production of a new mould, whereas with the 3DP technologies, this will only require the modification of the 3D design.

If the three 3DP technologies (FDM, SLA and SLS) were to be compared to one another, SLS can be considered to be more cost-effective than FDM and SLA (Figure 3). Whilst this contradicts with what has been claimed in previous pharmaceutical papers, wherein the prevalent use of FDM was correlated with its apparent cost-effectiveness [31], it is quite interesting to point out that in these calculations, the majority of the SLS cost accounts for the feedstock material. However, this is based on commercial feedstock and in pharmaceutical practice these numbers will differ inherently. This is because the feedstock utilised for SLS and FDM is identical and constitute a powder form of pharmaceutical grade excipients. In fact, the FDM feedstock will require further processing to produce filaments (e.g. the use of a hot melt extruder), which will further increase the cost of production.

As shown in Figure 3, as the number of units increased, the injection moulding curve plateaued out at about 12,000 units, yielding a cost similar to that of SLS. This is because the gained profit starts to compensate for the added cost of the machinery utilised for moulds production. Arguably, it can be concluded that whilst 3DP would serve as an ideal solution for customised production on a small scale (e.g. on-demand dispensing of personalised therapy in a pharmacy or clinic), for large-scale production, conventional production technologies (such as, tableting machines or injection moulding) would still remain superior. 
Insert figure 3.

\subsection{Economical and Logistical Benefits of 3D Printing Pharmaceuticals}

3DP is forecast to become the single biggest disruptive technology to the global industry since assembly lines were introduced in the late $20^{\text {th }}$ century [32]. Within the pharmaceutical industry, the promise of 3DP includes transitioning tablet production from centralised towards decentralised facilities (e.g. within the clinic, local pharmacies or even in the patient's home). As such, 3DP could likely help move away from traditional mass manufacture, towards mass customisation or personalisation $[32,33]$.

Decentralising pharmaceutical manufacture could provide three main benefits. First, the length and cost of transport and storage of pharmaceuticals could be reduced. In 2017, the global pharmaceutical industry was forecasted to be worth $\$ 1.2$ trillion, of which products worth $\$ 283$ billion required refrigerated storage and transport (2017 Cold Chain Outlook, http://pharmaceuticalcommerce.com/brand-marketing-communications/2017cold-chain-outlook/; 2017, [accessed 06 February 2018]; Pharmaceutical cold chain logistics is a $\$ 13.4$-billion global industry, http://pharmaceuticalcommerce.com/supply-chain-logistics/pharmaceuticalcold-chain-logistics-13-4-billion-global-industry/; 2017, [accessed 06 February 2018]). An alternative to such costly procedures could involve printlet or medical device designs being digitally created, sent across the globe electronically and printed on demand in a nearby clinical setting. As such, 3DPcould reduce carbon footprint by reducing fuel consumption associated with transport and avoiding the need for energy intensive storage conditions and manufacturing processes, such as injection moulding [34].

Second, it could offer a greater proximity to consumers, enabling quick and real-time responses to patient and market needs [35]. Local 3DP would be highly suited to the production of small batches of customised formulations. Examples include medicines that have narrow therapeutic windows that 
require exact dosing [36], or for complex formulations such as 'polypills' $[37,38]$ and those with unique geometries $[19,20,39,40]$. 3DP could also be used to produce personalised objects that could be tailored to a patient's body and requirements [41]. For instance, a customised sternum and partial ribcage were successfully 3D printed and implanted into a cancer patient, wherein titanium was combined with a porous polyethylene material that eases the incorporation of tissue material, while maintaining a bone-like structure (Csiro. NYC patient receives aussie-made 3D-printed sternum and rib cage transplant, https://www.csiro.au/en/News/News-releases/2017/NYCpatient-recieves-aussie-made-3d-printed-sternum; 2018 [accessed 18 April 2018]).

The concept of digital dispensing using 3DP could also have applications in hard-to-reach areas, such as within disaster zones, third world countries or even in space $[35,42]$. In 2017, the first medical supplies were 3D printed in space, whereby custom-fitted hand splint models were both designed and printed outside of Earth (Saunders, S., Astronauts 3D Print the First Medical Supplies in Space, Which Can Also Teach Us More About Healthcare on Earth, https://3dprint.com/162241/3d-print-medical-supplies-in-space/; 2017 [accessed 04 April 2018]). In-space manufacturing could also be applied to medicines, giving astronauts greater flexibility and autonomy when dealing with unexpected medical needs within deep space crewed missions.

Third, as 3DP enables a precise spatial control over the deposition of materials, a reduction in the amounts of API and excipients needed could be achieved [34]. This concept could benefit high cost medicines, such as 'orphan drugs' that are developed for rare diseases (affecting less than 1 in 2000 people in Europe) [43]. Due to the relatively small patient populations treated, costs of orphan drugs can be extremely high, placing financial burdens on patients and healthcare systems. For example, the cost of ivacaftor for cystic fibrosis is in excess of $\$ 290,000$ per patient per year [44]. In this case, 3DP could be utilised to limit waste compared to conventional technologies and hence reduce costs of development and dosing. 
Despite these benefits, it is clear that moving oral dosage form production towards local production (i.e. within clinical settings or at the patient's home) could raise a number of regulatory, legal and ethical considerations [45]. In particular, the introduction of distributed manufacturing using 3DP will bring about new challenges, such as issues surrounding data security, raw material storage and transport, quality control and risk of counterfeit production [46]. Moreover, the technical aspects of the 3DP process are yet to be well understood. For example, the reproducibility and accuracy of dosing (critical for narrow therapeutic index drugs) may be impacted by print resolution or homogeneity of mixing. In these instances, the use of technologies that provide high resolution (i.e. SLS and SLA [31]), as well as the development of on-site quality control methods that are suitable for the 3DP process may be required to ensure safety [7].

Current mass manufacturing facilities are governed by well-established good manufacturing practice (GMP) requirements to ensure patient and operator safety [47]. It is clear that new regulatory advice and guidance will be required before integration. Progress has already been made for additively manufactured medical devices, whereby the FDA released guidance detailing the technical considerations for such processes [48]. However, for 3DP of oral dosage forms, no such guidance has been released. As such, our research group at University College London (UCL) has initiated discussions with regulatory bodies to further advance the technology for this application.

From an economical perspective, it is likely that the production of high-volume and low-added value pharmaceuticals will remain more efficient in centralised manufacturing hubs. This is because the economies of scale of 3DP will likely never reach the same level as mass production [49]. However, there is value in scaling up 3DP processes, wherein personalisation is a requirement (e.g. implants and prosthesis). For example, several major manufacturers use 3DP to produce hearing aids in fairly large volumes ( 1000 devices/day), each being unique in shape and size [49]. Theoretically, similar principles could be applied to pharmaceuticals, whereby formulation shape, size, dosage and drug content could be adapted to suit the patient therapeutic needs and 
preferences. As such, this could facilitate patient autonomy in the treatment pathway, leading to increased medication adherence and therapeutic outcomes, as well as reduced wastage.

\subsection{Conclusion}

We stand on the brink of a revolution, where novel technologies such as 3DP are likely to cause a paradigm shift in pharmaceutical manufacture and supply. To date, the long-term benefits of this technology have been forecasted to lie within personalised medicines, leaving a wide range of opportunities underexplored. Indeed, 3DP could also provide many other advantages, ranging from applications in drug discovery, formulation manufacturing processes, global supply and logistics. In the future, 3DP could be used as a digital dispensing tool, supporting operations in hard-to-reach areas such as disaster zones and even within space. Indeed, the adoption of this highly disruptive technology will likely reshape the way that we design, manufacture and use medicines.

\subsection{Acknowledgements}

The authors thank the Engineering and Physical Sciences Research Council (EPSRC), UK for their partial financial support (EP/L01646X).

$[50][51] \quad[6,25,45,52,53] \quad[54] \quad[29,55] \quad[29,56,57] \quad[58] \quad[40,59] \quad[16,17,20,21,28,31,37-39,60] \quad[23]$ 


\subsection{References}

1 Rostow, W.W. (1990) The stages of economic growth: A noncommunist manifesto, Cambridge university press

2 Atkeson, A. and Kehoe, P.J. (2001) The Transition to a New Economy After the Second Industrial Revolution. National Bureau of Economic Research Working Paper Series No. 8676

$3 \quad$ Zhou, K. et al. (2015) Industry 4.0: Towards future industrial opportunities and challenges. In 2015 12th International Conference on Fuzzy Systems and Knowledge Discovery (FSKD), pp. 2147-2152

4 Rajkumar, R. et al. (2010) Cyber-physical systems: the next computing revolution. In Proceedings of the 47th Design Automation Conference, pp. 731-736, ACM

$5 \quad$ Campbell, T. et al. (2011) Could 3D printing change the world. Technologies, Potential, and Implications of Additive Manufacturing, Atlantic Council, Washington, DC

6 Barnatt, C. (2016) 3D Printing: Third Edition, CreateSpace Independent Publishing Platform

7 Trenfield, S.J. et al. (2018) 3D Printing Pharmaceuticals: Drug Development to Frontline Care. Trends in Pharmacological Sciences 39 (5), 440-451

8 Wong, C.H. et al. (2018) Estimation of clinical trial success rates and related parameters. Biostatistics kxx069, 1-14

$9 \quad$ Kitson, P.J. et al. (2016) The digital code driven autonomous synthesis of ibuprofen automated in a 3D-printer-based robot. Beilstein Journal of Organic Chemistry 12, 2776-2783

10 Kitson, P.J. et al. (2018) Digitization of multistep organic synthesis in reactionware for on-demand pharmaceuticals. Science 359 (6373), 314

11 Hornung, C.H. (2018) The art of manufacturing molecules. Science 359 (6373), 273-274

12 Halterman, T. (2014) Lee Cronin's 3D Printed Reactionware.

13 Lind, J.U. et al. (2016) Instrumented cardiac microphysiological devices via multimaterial three-dimensional printing. Nature Materials 16, 303 
14 Costello, C.M. et al. (2017) Microscale Bioreactors for in situ characterization of Gl epithelial cell physiology. Scientific Reports 7 (1), 12515

15 Hatton, G.B. et al. (2015) Animal Farm: Considerations in Animal Gastrointestinal Physiology and Relevance to Drug Delivery in Humans. Journal of Pharmaceutical Sciences 104 (9), 2747-2776

16 Genina, N. et al. (2017) Anti-tuberculosis drug combination for controlled oral delivery using 3D printed compartmental dosage forms: From drug product design to in vivo testing. Journal of Controlled Release 268 (Supplement C), 40-48

17 Goyanes, A. et al. (2018) PET/CT imaging of 3D printed devices in the gastrointestinal tract of rodents. Int J Pharm 536 (1), 158-164

$18 \mathrm{Ku}, \mathrm{M} . S$. and Dulin, W. (2012) A biopharmaceutical classificationbased Right-First-Time formulation approach to reduce human pharmacokinetic variability and project cycle time from First-In-Human to clinical Proof-Of-Concept. Pharm Dev Technol 17 (3), 285-302

19 Goyanes, A. et al. (2015) Effect of geometry on drug release from 3D printed tablets. Int J Pharm 494 (2), 657-663

20 Goyanes, A. et al. (2017) Patient acceptability of 3D printed medicines. International Journal of Pharmaceutics 530 (1), 71-78

21 Goyanes, A. et al. (2014) Fused-filament 3D printing (3DP) for fabrication of tablets. International Journal of Pharmaceutics 476 (1-2), 88-92

22 ASTM International. (2016) Standard Guidelines for Design for Additive Manufacturing. In Section 3: Terminology

23 Varotsis, A.B. (2018) Injection Molding: Manufacturing Technology Explained. (Vol. 2018)

24 Oskui, S.M. et al. (2016) Assessing and Reducing the Toxicity of 3DPrinted Parts. Environmental Science \& Technology Letters 3 (1), 1-6

25 Bloomquist, C.J. et al. (2018) Controlling release from 3D printed medical devices using CLIP and drug-loaded liquid resins. Journal of Controlled Release 278, 9-23

26 Yu, D.G. et al. (2009) Novel oral fast-disintegrating drug delivery devices with predefined inner structure fabricated by Three- 
Dimensional Printing. Journal of Pharmacy and Pharmacology 61 (3), 323-329

27 Goyanes, A. et al. (2015) 3D printing of modified-release aminosalicylate (4-ASA and 5-ASA) tablets. Eur J Pharm Biopharm 89, 157-162

28 Khaled, S.A. et al. (2014) Desktop 3D printing of controlled release pharmaceutical bilayer tablets. International Journal of Pharmaceutics 461 (1-2), 105-111

29 Goole, J. and Amighi, K. (2016) 3D printing in pharmaceutics: A new tool for designing customized drug delivery systems. Int J Pharm 499 (1-2), 376-394

30 Hopkinson, N. and Dicknes, P. (2003) Analysis of rapid manufacturing-using layer manufacturing processes for production. Proceedings of the Institution of Mechanical Engineers, Part C: Journal of Mechanical Engineering Science 217 (1), 31-39

31 Alhnan, M.A. et al. (2016) Emergence of 3D Printed Dosage Forms: Opportunities and Challenges. Pharm Res 33 (8), 1817-1832

32 Janssen, R.B., I; Moolenburgh, E; Posthumus, B. (2014) TNO: The impact of 3-D printing on supply chain management. .

33 Boon, W. and van Wee, B. (2017) Influence of 3D printing on transport: a theory and experts judgment based conceptual model. Transport Reviews, 1-20

34 Chen, D. et al. (2015) Direct digital manufacturing: definition, evolution, and sustainability implications. Journal of Cleaner Production 107, 615625

35 Manners-Bell, J.L., K. (2014) The Implications of 3D Printing for the Global Logistics Industry.

36 Vuddanda, P.R. et al. (2018) Personalisation of warfarin therapy using thermal ink-jet printing. European Journal of Pharmaceutical Sciences 117, 80-87

37 Khaled, S.A. et al. (2015) 3D printing of tablets containing multiple drugs with defined release profiles. International Journal of Pharmaceutics 494 (2), 643-650 
38 Khaled, S.A. et al. (2015) 3D printing of five-in-one dose combination polypill with defined immediate and sustained release profiles. Journal of Controlled Release 217, 308-314

39 Sadia, M. et al. (2018) Channelled tablets: An innovative approach to accelerating drug release from 3D printed tablets. Journal of Controlled Release 269, 355-363

40 Kyobula, M. et al. (2017) 3D inkjet printing of tablets exploiting bespoke complex geometries for controlled and tuneable drug release. J Control Release 261, 207-215

41 Goyanes, A. et al. (2016) 3D scanning and 3D printing as innovative technologies for fabricating personalized topical drug delivery systems. J Control Release 234, 41-48

42 Norman, J. et al. (2017) A new chapter in pharmaceutical manufacturing: 3D-printed drug products. Advanced Drug Delivery Reviews 108, 39-50

43 Downing, J. (2018) Cycle Pharmaceuticals to use 3D printing to develop 'orphan drugs'. In Cambridge Independent

44 Lancet. (2015) Reducing the cost of rare disease drugs. The Lancet 385 (9970), 746

45 Zema, L. et al. (2017) Three-Dimensional Printing of Medicinal Products and the Challenge of Personalized Therapy. J Pharm Sci 106 (7), 1697-1705

46 Kietzmann, J. et al. (2015) Disruptions, decisions, and destinations: Enter the age of 3-D printing and additive manufacturing. Business Horizons 58 (2), 209-215

$47 \quad \mathrm{ICH}$. (2000) Good Manufacturing Practice Guide for Active Pharmaceutical Ingredients. (ICH Harmonised Tripartite Guideline, ed.)

48 FDA. (2016) Technical Considerations for Additive Manufactured Devices.

49 Ye, M. (2015) The Impact of 3D Printing on the World Container Transport. In Delft University of Technology

50 Aniwaa. (2018) 3D printers comparison. (Vol. 2018)

51 AMRG. (2018) About additive manufacturing. (Vol. 2018) 
52 Wang, J. et al. (2016) Stereolithographic (SLA) 3D printing of oral modified-release dosage forms. International Journal of Pharmaceutics 503 (1-2), 207-212

53 Martinez, P.R. et al. (2017) Fabrication of drug-loaded hydrogels with stereolithographic 3D printing. Int J Pharm 532 (1), 313-317

54 Gibson, I. et al. (2015) Sheet Lamination Processes. In Additive Manufacturing Technologies: 3D Printing, Rapid Prototyping, and Direct Digital Manufacturing (Gibson, I. et al., eds.), pp. 219-244, Springer New York

55 Içten, E. et al. Dropwise Additive Manufacturing of Pharmaceutical Products for Melt-Based Dosage Forms. Journal of Pharmaceutical Sciences 104 (5), 1641-1649

56 Fina, F. et al. (2017) Selective laser sintering (SLS) 3D printing of medicines. International Journal of Pharmaceutics 529 (1), 285-293

57 Fina, F. et al. (2018) Fabricating 3D printed orally disintegrating printlets using selective laser sintering. International Journal of Pharmaceutics 541 (1), 101-107

58 Gibson, I. et al. (2015) Directed Energy Deposition Processes. In Additive Manufacturing Technologies: 3D Printing, Rapid Prototyping, and Direct Digital Manufacturing (Gibson, I. et al., eds.), pp. 245-268, Springer New York

59 Gibson, I. et al. (2015) Binder Jetting. In Additive Manufacturing Technologies: 3D Printing, Rapid Prototyping, and Direct Digital Manufacturing (Gibson, I. et al., eds.), pp. 205-218, Springer New York

60 Beck, R.C.R. et al. (2017) 3D printed tablets loaded with polymeric nanocapsules: An innovative approach to produce customized drug delivery systems. International Journal of Pharmaceutics 528 (1), 268279 


\section{List of Figures:}

Figure 1: Graphical representation of the opportunities in which 3DP can be implemented.

Figure 2: Graphical representation of the different 3DP technologies. SLA: Stereolithography; DLP: Direct light processing; CLIP: Continuous liquid interface production; BJ: Binder jetting; SLS: Selective laser sintering; DMLS/ SLM: Direct metal laser sintering/selective laser melting; MJF: Material jet fusion; EBM: Electron beam melting; NPJ: Nanoparticle jetting; MJ: Material jetting; DOD: Drop-on-demand; LENS: Laser engineering net shape; EBAM: Electron beam additive manufacturing; LOM: Laminated object manufacturing; UAM: Ultrasonic additive manufacturing; FDM: Fused deposition modelling; SSE: Semi-solid extrusion.

Figure 3: Comparison chart showing the production cost of an object when manufactured using injection moulding, stereolithography, fused deposition modelling and selective laser sintering. (Reprinted with permission from [30]) 


\section{List of Tables:}

Table 1: An overview on the features associated with each additive manufacturing technology. SLA: Stereolithography; DLP: Direct light processing; CLIP: Continuous liquid interface production; BJ: Binder jetting; SLS: Selective laser sintering; DMLS/ SLM: Direct metal laser sintering/selective laser melting; MJF: Material jet fusion; EBM: Electron beam melting; NPJ: Nanoparticle jetting; MJ: Material jetting; DOD: Drop-ondemand; LENS: Laser engineering net shape; EBAM: Electron beam additive manufacturing; LOM: Laminated object manufacturing; UAM: Ultrasonic additive manufacturing; FDM: Fused deposition modelling; SSE: Semi-solid extrusion. 